Mappemonde

Revue trimestrielle sur l'image géographique et les formes du territoire

$129 \mid 2020$

Varia

\title{
La Baltique, un espace d'intégration, mais aussi de concurrence et de rivalités
}

\section{Arnaud Serry}

\section{OpenEdition}

Journals

Édition électronique

URL : http://journals.openedition.org/mappemonde/4521

DOI : $10.4000 /$ mappemonde.4521

ISSN : 1769-7298

\section{Éditeur}

UMR ESPACE

\section{Référence électronique}

Arnaud Serry, «La Baltique, un espace d'intégration, mais aussi de concurrence et de rivalités »,

Mappemonde [En ligne], 129 | 2020, mis en ligne le 10 novembre 2020, consulté le 26 novembre 2020.

URL : http://journals.openedition.org/mappemonde/4521 ; DOI : https://doi.org/10.4000/

mappemonde.4521

Ce document a été généré automatiquement le 26 novembre 2020.

\section{cc) (1) (ㅇ)}

La revue Mappemonde est mise à disposition selon les termes de la Licence Creative Commons Attribution - Pas d'Utilisation Commerciale - Partage dans les Mêmes Conditions 4.0 International. 


\title{
La Baltique, un espace d'intégration, mais aussi de concurrence et de rivalités
}

\author{
Arnaud Serry
}

1 En 2019 paraissait l'article de Mappemonde intitulé «Trente ans d'images dans Mappemonde ", écrit par Clarisse Didelon-Loiseau et Hervé Théry. Les auteurs y faisaient le constat que l'Europe et le bassin méditerranéen y étaient largement couverts, à l'exception notable des pays baltes. Ayant pour origine ce constat, ce dossier a notamment pour objectif de pallier cette carence, d'autant plus que l'espace baltique a connu depuis une trentaine d'années des bouleversements politiques, géopolitiques et économiques majeurs.

2 Environ 65 millions de personnes vivent à moins de 300 kilomètres des rives de la mer Baltique, mais cette région baltique est diverse : le long des côtes, on parle neuf langues différentes, les traditions religieuses varient d'un territoire à l'autre, les niveaux de développement économique sont relativement contrastés. Nulle part ailleurs en Europe il n'y a eu, ces dernières années, autant de changements politiques et économiques. L'éclatement du bloc de l'Est a, dans une certaine mesure, unifié une zone traversée il y a peu par le rideau de fer dont la chute est souvent perçue comme un retour à l'unité de la mer Baltique. Le caractère maritime de cet espace est régulièrement cité comme révélateur de cette unité, comme le résume très clairement Jacques Marcadon: «Le trait d'union entre les pays de la Baltique est la mer » (Marcadon, 1998). La région n'en garde pas moins ses spécificités.

De manière récurrente, la mer Baltique est considérée, décrite, comme un facteur de contact et de rapprochement entre les populations, les États et les cultures qui la bordent. Bien qu'elles présentent des différences marquées, les rives baltiques forment un espace qui vient d'achever une reconfiguration majeure depuis le début des années 1990 : émancipation des États baltes de l'influence russe et renforcement de l'Union européenne dans la région en sont deux symboles forts. 
4 Si cette mer a été traversée à différentes époques et jusqu'à aujourd'hui par des frontières est-ouest, nord-sud ou plus complexes, entre religions, empires ou idéologies, ce dossier interroge l'unité et l'hétérogénéité contemporaine des espaces et territoires qui bordent la mer Baltique, sur différents plans (environnemental, culturel, économique, géopolitique...). D'autant plus que l'annexion de la Crimée par la Russie en 2014 a replacé l'espace baltique au centre des débats géopolitiques. Les recherches montrent en effet l'apparition de trois discours présentant la Baltique comme un espace environnementalement en danger, comme le théâtre d'une nouvelle guerre froide et comme une interface entre la Russie et l'Europe. Aussi, l'espace baltique, symbole de coopération, baigné dans un discours sur le retour du lac baltique au début de la décennie 1990, est désormais le théâtre de stratégies de plus en plus différenciées.

Ces stratégies spécifiques sont particulièrement présentes dans le monde du shipping et dans les stratégies portuaires. C'est ce que relèvent Arnaud Serry et Lilian Loubet dans un article consacré à la gouvernance portuaire comme enjeu de développement en Baltique orientale (https://journals.openedition.org/mappemonde/4828). La réorganisation $\mathrm{du}$ transport maritime illustre en effet les profondes et rapides mutations de la région, son positionnement comme interface ou encore la différenciation des trajectoires. Particulièrement, les évolutions en matière de gouvernance portuaire, qui constituent un enjeu majeur de développement territorial, incarnent des dynamiques fortes de glissement d'une certaine complémentarité à une concurrence féroce liée à l'intégration des ports dans le système maritime et portuaire mondial.

6 Par ailleurs, sur les rives baltiques, les enjeux énergétiques pèsent également lourd dans les rapports entre les États, notamment ceux avec Moscou. Les infrastructures des systèmes énergétiques, comme les gazoducs Nord Stream, prennent un rôle singulier dans cette construction de la Baltique comme nouveau lieu géopolitique signifiant dans les relations internationales. L'énergie nucléaire, qui connaît un développement singulier dans l'espace baltique au regard de son évolution dans le reste du continent, questionne les représentations géopolitiques concurrentes. Ils sont aujourd'hui mis en lumière par Teva Meyer au regard des débats suédois sur la centrale finlandaise de Pyhäjoki (https://journals.openedition.org/mappemonde/4748).

7 Le développement touristique est une réalité qui touche toutes les rives baltiques. Les acteurs multiplient les stratégies afin d'attirer investisseurs et touristes, quitte à jouer le jeu de la concurrence. Dans ce contexte de concurrence et d'intégration baltique, le tourisme apparaît alors comme un enjeu pour de nombreuses régions. En Allemagne du Nord, la plupart des villes étant également des ports, ce développement autour des grands corridors maritimes exacerbe les rivalités. Lauriane Létocart évoque ces dynamiques touristiques en s'intéressant au cas du Mecklembourg-PoméranieOccidentale, un littoral touristique entre réhabilitation et ancrage dans une aire allemande et baltique. Les dynamiques touristiques, en s'adaptant à des reconfigurations multiscalaires, mettent en avant des stratégies d'acteurs visant à se positionner au sein de l'espace baltique. Étudiées dans ce dossier à l'échelle du littoral du Mecklembourg-Poméranie-Occidentale celles-ci révèlent une co-construction entre les aménagements, les pratiques et les stratégies politiques (à paraître).

8 Géopolitiquement, la mer Baltique a inlassablement été un espace complexe: ligue hanséatique, rideau de fer ou antagonisme contemporain entre la Russie et l'OTAN en sont quelques exemples. Ainsi, ce début de $\mathrm{XXI}^{\mathrm{e}}$ siècle a aussi vu resurgir dans la région 
des questions de sécurité dure, évacuées après la chute du Rideau de fer, en lien avec les craintes de menace russe. Nicolas Escach aborde cette question à travers les vulnérabilités et recompositions baltiques et les conséquences d'une géopolitique insidieuse. Il met en avant une certaine multiplication des fragilisations et questionne la vulnérabilité des territoires à toutes les échelles menaçant ou altérant l'interterritorialité si souvent associée à l'espace baltique (à paraître).

\section{BIBLIOGRAPHIE}

MARCADON J. (1998). «L'évolution récente de l'activité maritime et portuaire dans les pays de la Baltique ». Bulletin de l'Association de géographes français : la nouvelle Europe médiane, p. 428-436.

INDEX

Thèmes : La Baltique. Un espace d'intégration mais aussi de concurrence et de rivalités 